\title{
Sequence analysis of the L protein of the Ebola 2014 outbreak: Insight into conserved regions and mutations
}

\author{
GOHAR AYUB $^{1}$ and YASIR WAHEED ${ }^{1,2}$ \\ ${ }^{1}$ Department of Health Biotechnology, Atta-ur-Rahman School of Applied Biosciences, \\ National University of Sciences and Technology, Islamabad 44000; ${ }^{2}$ Multidisciplinary Labs, \\ Foundation University Medical College, Foundation University Islamabad, \\ Defence Housing Authority Phase I, Islamabad 46000, Pakistan \\ Received February 17, 2015; Accepted December 11, 2015
}

DOI: $10.3892 / \mathrm{mmr} .2016 .5145$

\begin{abstract}
The 2014 Ebola outbreak was one of the largest that have occurred; it started in Guinea and spread to Nigeria, Liberia and Sierra Leone. Phylogenetic analysis of the current virus species indicated that this outbreak is the result of a divergent lineage of the Zaire ebolavirus. The L protein of Ebola virus (EBOV) is the catalytic subunit of the RNA-dependent RNA polymerase complex, which, with VP35, is key for the replication and transcription of viral RNA. Earlier sequence analysis demonstrated that the $\mathrm{L}$ protein of all non-segmented negative-sense (NNS) RNA viruses consists of six domains containing conserved functional motifs. The aim of the present study was to analyze the presence of these motifs in 2014 EBOV isolates, highlight their function and how they may contribute to the overall pathogenicity of the isolates. For this purpose, $812014 \mathrm{EBOV}$ L protein sequences were aligned with 475 other NNS RNA viruses, including Paramyxoviridae and Rhabdoviridae viruses. Phylogenetic analysis of all EBOV outbreak $\mathrm{L}$ protein sequences was also performed. Analysis of the amino acid substitutions in the 2014 EBOV outbreak was conducted using sequence analysis. The alignment demonstrated the presence of previously conserved motifs in the 2014 EBOV isolates and novel residues. Notably, all the mutations identified in the $2014 \mathrm{EBOV}$ isolates were tolerant, they were pathogenic with certain examples occurring within previously determined functional conserved motifs, possibly altering viral pathogenicity, replication and virulence. The phylogenetic analysis demonstrated that all sequences with the exception of the 2014 EBOV sequences were clustered together. The 2014 EBOV outbreak has acquired a great
\end{abstract}

Correspondence to: Dr Yasir Waheed, Multidisciplinary Labs, Foundation University Medical College, Foundation University Islamabad, Jinnah Avenue, Defence Housing Authority Phase I, Islamabad 46000, Pakistan

E-mail: yasir_waheed_199@hotmail.com

Key words: Ebola virus, L protein, phylogenetic analysis, sequence comparison number of mutations, which may explain the reasons behind this unprecedented outbreak. Certain residues critical to the function of the polymerase remain conserved and may be targets for the development of antiviral therapeutic agents.

\section{Introduction}

Filoviruses are responsible for complex hemorrhagic fevers in humans and primates with case fatality rates of 50-90\% (1). The filovirus family belongs to the order Mononegavirales and is divided into two genera: Ebolavirus comprising five species, Zaire ebolavirus (EBOV), Taï Forest ebolavirus, Reston ebolavirus, Bundibugyo ebolavirus and Sudan ebolavirus; and Marburgvirus, which contains one species, Marburg marburgvirus. A third genus, Cuevavirus has also been recently proposed (2). Ebolaviruses have a non-segmented negative-sense (NNS) single-stranded RNA genome, a characteristic of the order, Mononegavirales, and most closely resembling the families Rhabdoviridae and Paramyxoviridae in its genetic assembly. The EBOV genome is $\sim 19,000$ base pairs in length and contains seven open reading frames that code for seven structural proteins in order, from $3^{\prime}$ to $5^{\prime}$, nucleoprotein (NP), VP35, VP40, glycoprotein (GP), VP30, VP24 and the L protein (3).

The $\mathrm{L}$ protein is 2,212 amino acids in length and is the largest protein encoded by the EBOV virus. It does not function independently but is part of an RNA-dependent RNA polymerase (RdRp) complex with the NP and viral transcription factors, VP30 and VP35. This complex is important in the replication and transcription of the viral genome. The $\mathrm{L}$ protein is hypothesized to serve as the major catalytic subunit of this complex (4). The majority of the data available on the structure and function of the EBOV L protein is theoretical and inadequate, relying on comparative analysis and data from the L proteins of other well-studied NNS RNA viruses of the order Mononegavirales rather than on experimental observations.

The current 2014 EBOV epidemic in West Africa is the largest ever reported Ebola epidemic across multiple West African countries with unprecedented transmissibility. There are no marketed antiviral therapeutic agents or vaccines against EBOV and the continued search for effective and safe therapeutic agents is required (5). RdRp of other RNA 


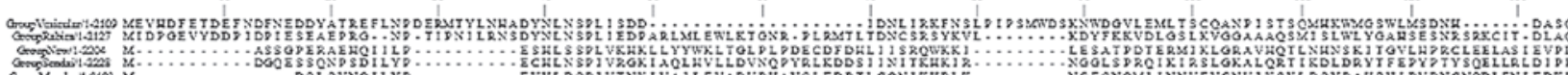

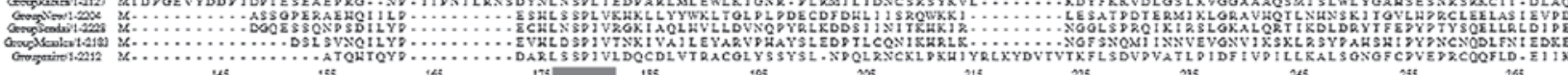

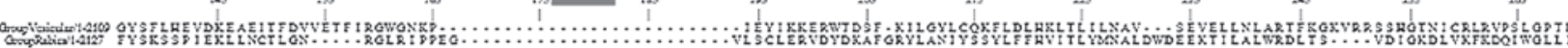

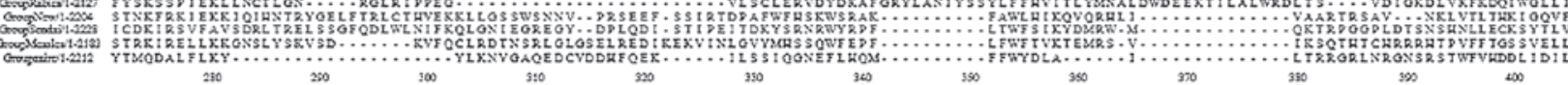

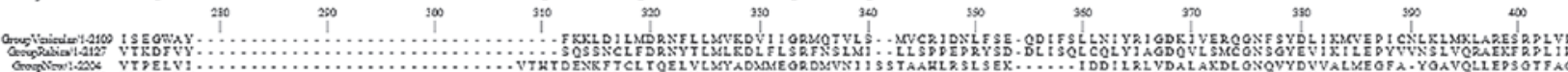

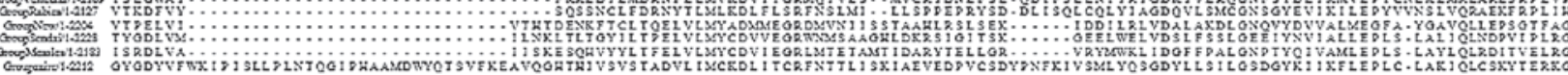

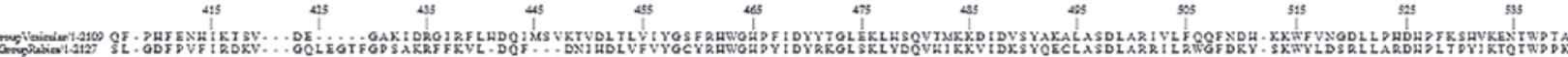

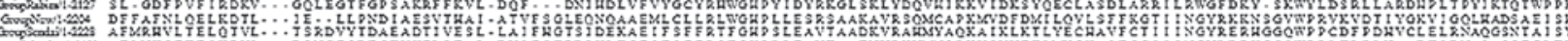

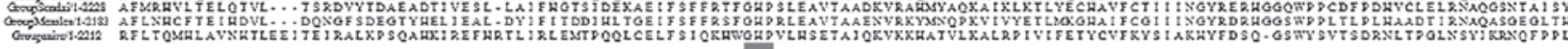

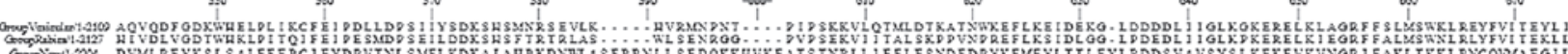

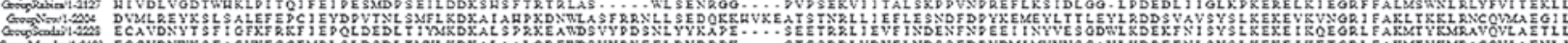

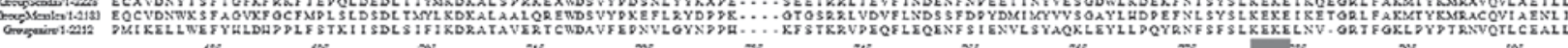

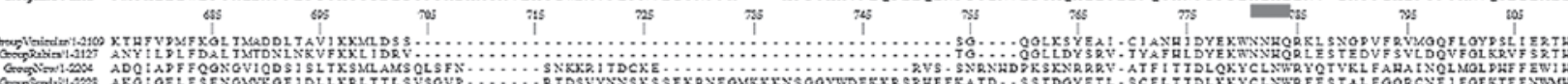

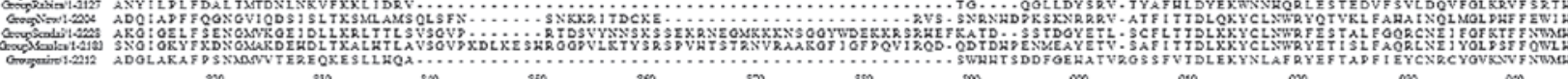

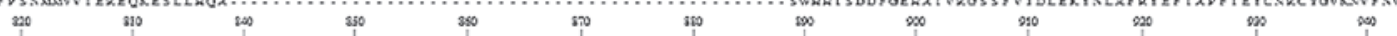

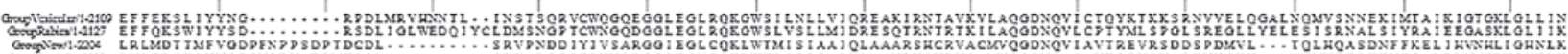

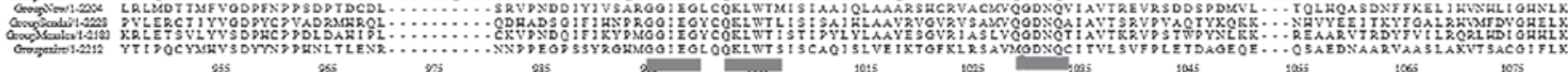

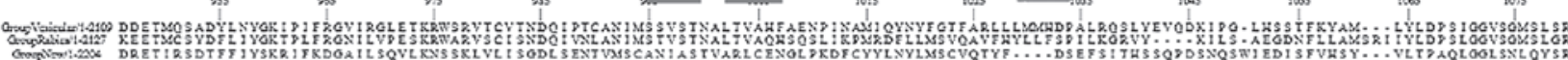

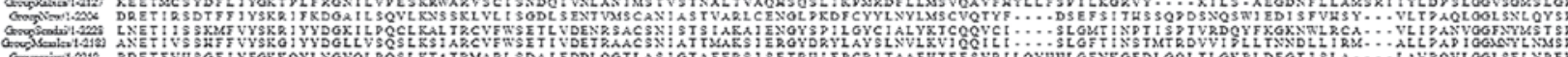

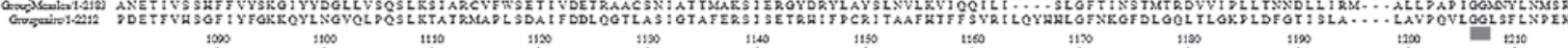

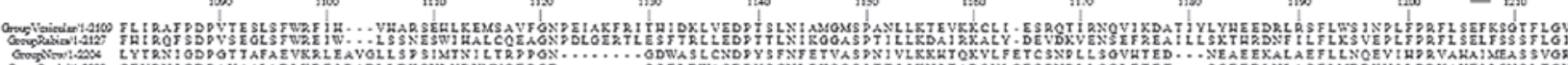

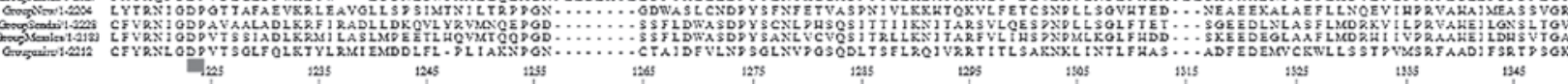

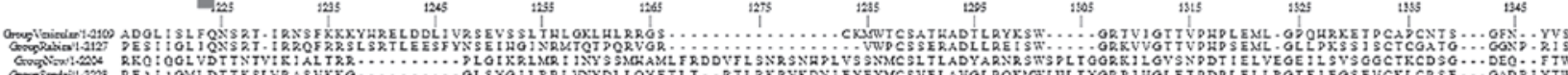

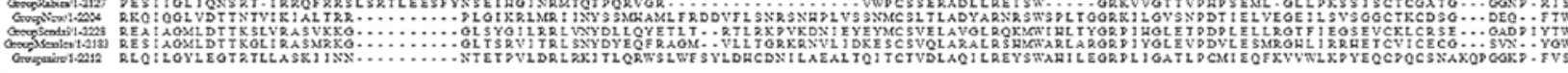

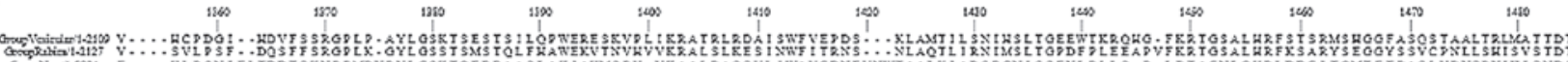

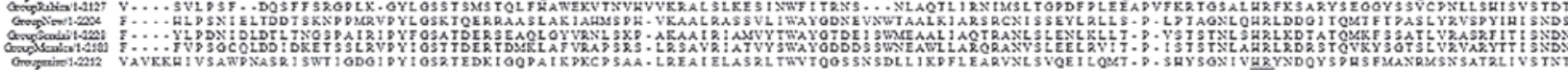

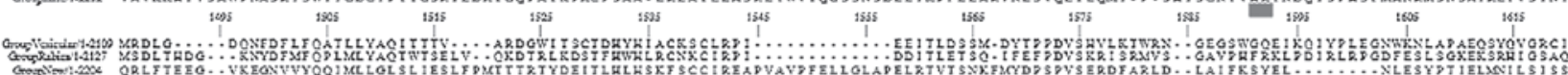

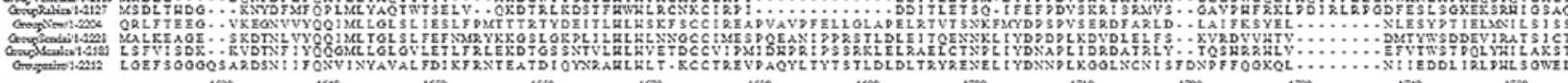

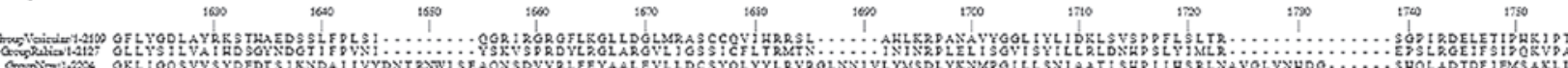

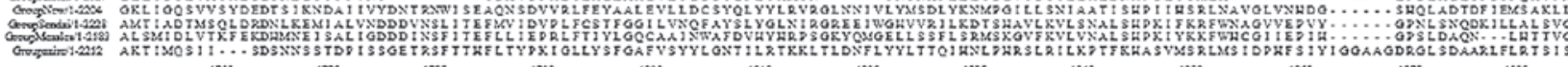

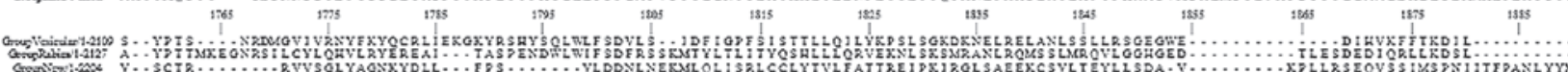

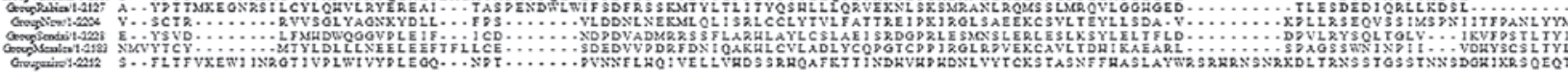

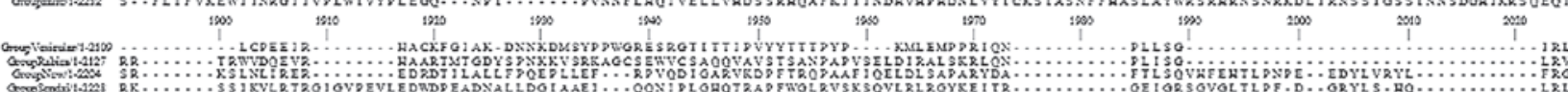

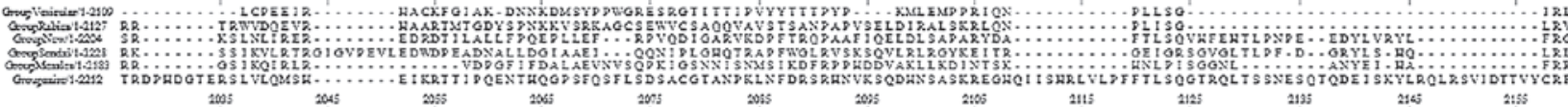

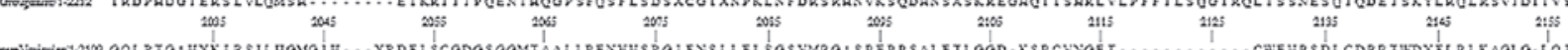

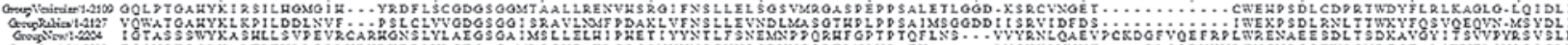

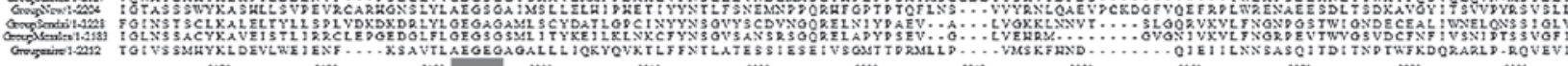

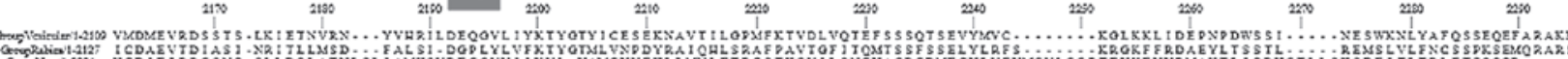

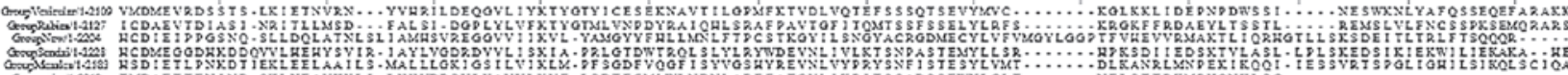

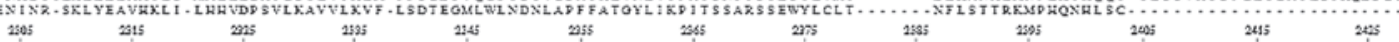

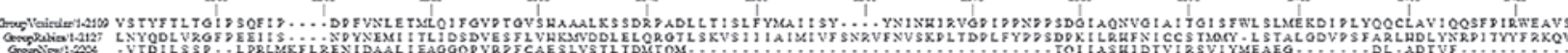

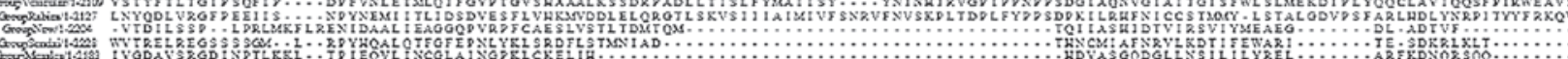

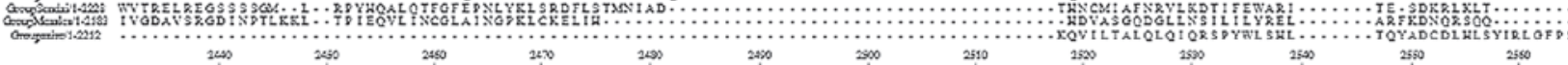

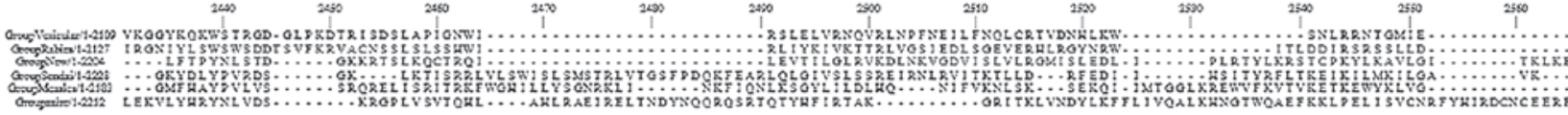

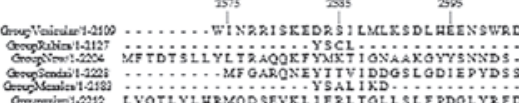

Figure 1. Amino acid sequence alignment of the L proteins of vesicular stomatitis virus, rabies virus, Newcastle disease virus, Sendai virus, measles virus and Zaire ebolavirus. Analysis was performed with the MAFFT program using the L-INS-i algorithm. The dashes represent gaps introduced to optimize the alignment. Motif/residues discussed in the text are highlighted in dark grey. Only a representative consensus sequence for each virus is presented. 


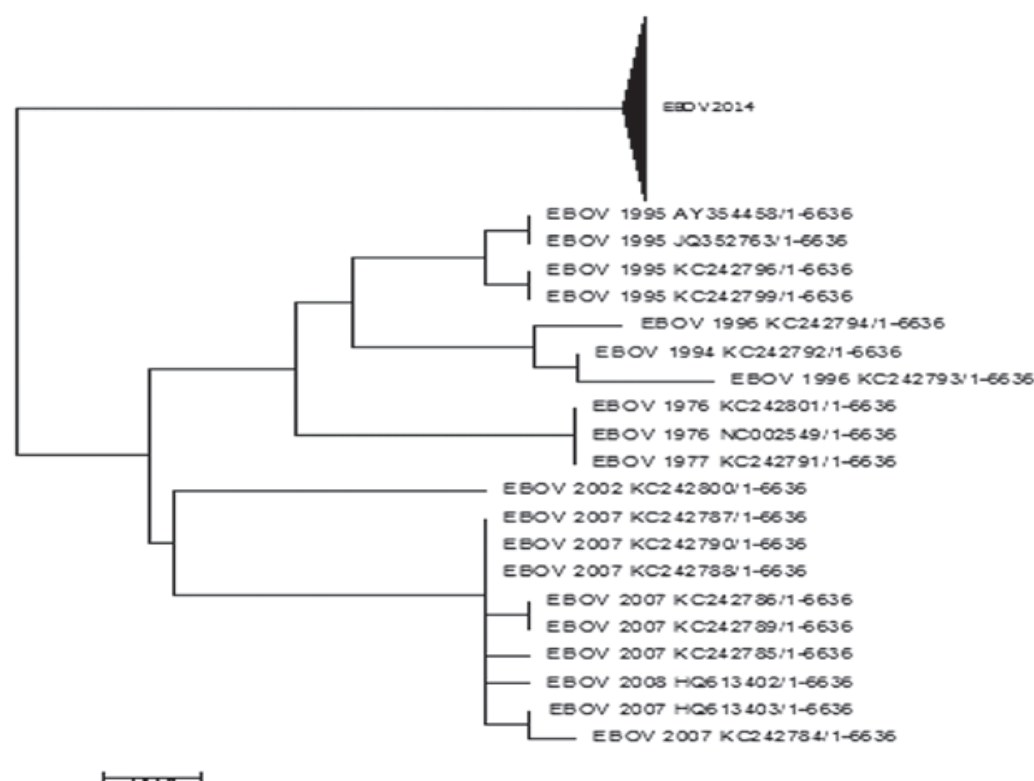

Figure 2. Phylogenetic analysis of EBOV species in different outbreaks. The phylogenetic analysis was inferred using the Neighbor-Joining method. The tree is drawn to scale, with branch lengths in the same units as those of the evolutionary distances used to infer the phylogenetic tree. The evolutionary distances were computed using the Poisson correction method and are in the units of the number of amino acid substitutions per site. The analysis involved 101 amino acid sequences. The EBOV 2014 sequences are presented as a single black triangle. There were a total of 2,172 positions in the final dataset. Evolutionary analyses were conducted in MEGAv.6.0.6. EBOV, Zaire ebolavirus.

viruses, such as hepatitis $\mathrm{C}$ virus, have been successful targets for the development of antiviral therapeutic agents and vaccines due to the presence of various highly conserved motifs and/or residues $(6,7)$. A previous study identified single nucleotide polymorphisms that distinguish the 2014 West African outbreak from previous outbreaks and demonstrated how the virus spread from Guinea to Sierra Leone in a matter of few months (8). The authors have identified 16 novel non-synonymous amino acid substitutions in the $\mathrm{L}$ protein that are present in the 2014 outbreak isolates (8).

In the present study, the 2014 EBOV L protein sequences were compared with other NNS RNA viruses, including Paramyxoviridae and Rhabdoviridae. The majority of previously reported L protein specific linear were conserved (9). Novel conserved regions that were not previously documented were also identified in the current study. Notably, all the mutations identified in the $2014 \mathrm{EBOV}$ isolates were tolerant, they were pathogenic with certain examples occurring within previously determined functional conserved motifs, possibly attenuating viral pathogenicity, replication and virulence. Phylogenetic analysis of $\mathrm{L}$ protein sequences from past EBOV outbreaks was also conducted and was consistent with previous studies.

\section{Materials and methods}

Sequence alignment. In order to align the sequences, 475 amino acid sequences of the $\mathrm{L}$ proteins belonging to the family Rhabdoviridae (vesicular stomatitis virus and rabies virus) and Paramyxoviridae (Sendai virus, Newcastle disease virus and measles virus) were randomly selected from the National Center for Biotechnology Information (NCBI) Protein Database (http://www.ncbi.nlm.nih.gov/protein). The sequences were analyzed in the Jalview Desktop Multiple
Alignment Editor (v.2.8.1) (10) with 81 EBOV L protein amino acid sequences from the 2014 outbreak, to draw a representative consensus sequence for each of the six viruses. The resulting consensus sequences of all six viruses were then aligned using the built-in MAFFT multiple sequence alignment program (v.7.205) (11) with the L-INS-i algorithm (12).

Phylogenetic tree construction. A phylogenetic tree of all 101 EBOV sequences reported to date (including the 2014 EBOV sequences) was also constructed in MEGA.v.6.06 (13), employing the Neighbor-Joining algorithm with the Poisson correction method (14). All 812014 EBOV sequences were used in constructing the tree; however, they are presented as a single group in the tree figure for clarity.

Amino acid substitution analysis. Furthermore, a standard prediction tool, SIFT Blink was used to predict and analyze the effect of the 16 amino acid substitutions on the function of the $\mathrm{L}$ protein (15).

\section{Results}

Consensus sequencing. In the present study, $475 \mathrm{~L}$ protein sequences of vesicular stomatitis virus, rabies virus, Newcastle disease virus, Sendai virus, measles virus and EBOV were extracted. The sequences were fed into the Jalview Desktop Multiple Alignment Editor (v.2.8.1) software to draw consensus sequences of the $\mathrm{L}$ protein of each virus. The consensus sequences of the $\mathrm{L}$ proteins were aligned to analyze the sequence variation (Fig. 1). Each row demonstrates a representative consensus sequence for each virus respectively. The dashes represent gaps introduced to optimize the alignment. The six-way alignment established that strong homologies exist over almost the entire length of the proteins. In addition, 
Table I. Amino acid differences observed in L protein between the 2014 and 2007 EBOV outbreak.

\begin{tabular}{lcc}
\hline Amino acid position & EBOV 2014 & EBOV 2007 \\
\hline 197 & L & M \\
202 & T & S \\
215 & T & A \\
337 & I & M \\
342 & P & S \\
346 & H & Q \\
692 & N & D \\
759 & G & D \\
1405 & Q & R \\
1607 & H & Q \\
1610 & F & L \\
1615 & N & S \\
1654 & D & Y \\
1656 & T & A \\
1658 & N & D \\
1673 & K & E \\
1685 & D & G \\
1690 & S & N \\
1729 & P & S \\
1753 & G & D \\
1774 & Q & K \\
1826 & N & S \\
1944 & H & Y \\
1951 & V & I \\
2059 & L & F \\
2085 & V & I \\
\hline & & \\
\hline & &
\end{tabular}

EBOV, Zaire ebolavirus.

the gaps introduced in order to maximize similarities are typically limited. The relative frequency of Gly (14.3 ) and Trp (3-9\%) residues among the invariant amino acids is markedly higher (2.5-3-fold) compared with their average abundance in the $\mathrm{L}$ proteins ( 4.5 and $5.5 \%$, respectively), signifying that these residues are particularly significant for the L protein structure and/or function, whereas basic and acidic residues also tend to be more strictly retained than hydrophobic ones. A similarity profile of the six-way alignment (Fig. 1) clearly revealed that conserved residues are not scattered randomly, but are clustered into six blocks of strong conservation (11) connected by variable regions of low conservation. The blocks of highest amino acid conservation (II-V) were located in the central region of the protein (positions 521-1397). In each block there were uninterrupted stretches of strictly or conservatively maintained amino acids, as highlighted in the figure. In particular, the highly conserved motifs within the EBOV share extended similarities with motifs found throughout all other RNA dependent polymerases. Thus, these may well constitute the active site for phosphodiester bond formation and/or template recognition of unsegmented negative-strand RNA virus polymerases.
Table II. Fixed amino acid substitutions observed in the L proteins of the 2014 EBOV isolates along with SIFT amino acid predictions.

\begin{tabular}{lccc}
\hline $\begin{array}{l}\text { Amino } \\
\text { acid position }\end{array}$ & $\begin{array}{c}\text { Ancestral } \\
\text { amino acid }\end{array}$ & $\begin{array}{c}\text { Substituted } \\
\text { amino acid }\end{array}$ & $\begin{array}{c}\text { SIFT amino } \\
\text { acid predictions }\end{array}$ \\
\hline 197 & M & L & Tolerated \\
215 & A & T & Tolerated \\
337 & M & I & Tolerated \\
346 & G & H & Tolerated \\
692 & A & A & Tolerated \\
1607 & G & H & Tolerated \\
1615 & S & A & Tolerated \\
1654 & T & A & Tolerated \\
1656 & A & T & Tolerated \\
1658 & A & A & Tolerated \\
1673 & G & L & Tolerated \\
1690 & A & S & Tolerated \\
1826 & S & A & Tolerated \\
1944 & T & H & Tolerated \\
1951 & I & V & Tolerated \\
2085 & I & V & Tolerated \\
\hline
\end{tabular}

EBOV, Zaire ebolavirus.

Phylogenetic analysis. The 2014 EBOV isolates were recovered from Sierra Leone and Guinea, countries with the worst outbreak of Ebola virus disease (EVD) in history. To determine the evolutionary relationship between these isolates with those of previous EBOV outbreaks, a phylogenetic analysis was conducted using the sequences of the $\mathrm{L}$ proteins as presented in Fig. 2. The phylogenetic analysis indicated that all but the 2014 EBOV sequences were clustered together. This suggests that previous outbreaks accumulated few mutations in the L protein and were more closely associated with each other as the majority of the previous outbreaks exhibit almost identical sequences (8). A sequence comparison (not shown) of the L protein from the 2014 EBOV outbreak and 2007 EBOV outbreak alone demonstrated 26 amino acid substitutions, confirming the high mutation rate of the virus (Table I) (8). The branch leading towards the 2014 EBOV outbreak is long compared with others as it is a divergent lineage that has accumulated multiple mutations.

Amino acid substitution analysis. Table II presents all 16 amino acid substitutions that, when compared with ancestral amino acid residues, were fixed within the 2014 EBOV outbreak according to the SIFT amino acid prediction of substitution. To assess the effect of a substitution, SIFT assumes that important positions in a protein sequence have been conserved throughout evolution and therefore substitutions at these positions may have an adverse effect on protein function. Thus, by using sequence homology, SIFT predicts the effects of all possible substitutions at each position in the protein sequence. All substitutions were tolerant in the present study; hence, it can be inferred that amino acid substitutions or insertions/deletions in these regions were unlikely to affect function. 


\section{Discussion}

Sequences of residues ranging in length from four to seven amino acids, clustered into six domains of strong conservation and separated by variable regions have been defined for the families, Rhabdoviridae and Paramyxoviridae $(9,16)$. These six domains were also observed to be conserved in previous EBOV L proteins compared with paramyxoviruses (17). Notably, these conserved regions, are not retained in the current 2014 EBOV isolates, except in the mutated regions. The domains are key for crucial activities conducted by the L proteins.

Three polymerase motifs, motif A (aa 646-665), motif B (aa 892-898) and motif C (aa 2,034-2,063) identified previously in other NNS viruses, including EBOV L proteins were also observed in the 2014 EBOV isolates, but with a number of mutations (17). The invariant tripeptide GHP motif (aa 464-466), recognized previously as a turn structure exposing the histidine residue, was identified to be conserved amongst all the viruses (9). This motif may potentially serve as a target for future antiviral therapeutic agents, and site-specific mutagenesis studies may further elucidate its function.

Another pentapeptide, QGDNQ (aa 894-898), part of motif B is conserved in all NNS viruses, excluding EBOV. This motif is crucial for RNA polymerase function and phosphodiester bond formation and/or template recognition. In EBOV, the first glycine residue is substituted for a methionine residue (18). This substitution is not specific to the 2014 EBOV isolates as it has been demonstrated across all other Ebolavirus species. This substitution was not key in the 2014 outbreak, however, its evolutionary role in the transcription and the replication process requires further elucidation. Furthermore, the GDN part of the sequence corresponds to the strictly conserved residues, GDD in other polymerases (18). It is therefore an ideal target for antiviral therapeutic agents.

The KEKE tetrapeptide (aa 646-649), part of motif A that is responsible for the positioning and binding of the RNA template, was observed to be conserved in all viruses, except vesicular stomatitis and rabies viruses. In Rhabdoviridae, the lysine residue was substituted for glutamic acid, consistent with previous studies indicating that filoviruses are more closely associated with the paramyxoviruses than to rhabdoviruses $(9,16,17)$.

Another pentapeptide motif GG(I/L)EG (aa 860-865) was also conserved in the alignment. EBOV contained an isoleucine rather than a leucine, further suggesting a closer association with the family Paramyxoviridae $(9,19)$. Two invariant dipeptides GG (aa 1,071-1,072) and DP (aa 1,088-1,089) were also conserved as reported previously (9).

The HR motif (aa 1,456-1,457), previously indicated to be important for the PRNTase activity of the L protein at the enzyme-pRNA intermediate formation step, was also observed to be conserved across all the virus sequences $(16,20)$.

Furthermore, the GXGXG pentapeptide (aa 2,057-2,061) part of motif $\mathrm{C}$ was demonstrated to be conserved in all members of the Rhabdoviridae family and two members of the Paramyxoviridae family (Sendai and measles) with slight inter-species variations in the $\mathrm{X}$ amino acids. This pentapeptide is hypothesized to be involved in mRNA capping via a 2'-O-ribose-methyltransferase action (21).
To the best of our knowledge, this is the first study to demonstrate that the $\mathrm{L}(\mathrm{N} / \mathrm{S}) \mathrm{SP}(\mathrm{L} / \mathrm{I}) \mathrm{V}$ motif (aa 41-46) is conserved across the viruses. However, the function of this motif remains to be elucidated. A QK $(\mathrm{G} / \mathrm{L}) \mathrm{W}(\mathrm{S} / \mathrm{T})$ motif (aa 867-871) was also observed to be conserved, the exact function of this motif remains to be determined. In addition, the pattern of conservation itself is notable, beyond the 1,540th amino acid, the conservation decreases to the 2,024th amino acid, following which there is another stretch of partially conserved residues to the 2,241th amino acid. Beyond this, there is a highly non-conserved region filled with large gaps. This demonstrates that the conserved residues predominantly lie in the mid-region of the protein, consistent with the results of a previous study (9).

Furthermore, the phylogenetic tree of the EBOV sequences is similar to trees drawn previously on the basis of GP and NP sequences, demonstrating the same pattern of clustering (22). Though the previously drawn trees did not take into account the latest 2014 sequences, the pattern of clustering remains the same, predominantly due to minimal mutations acquired by the viruses in previous outbreaks. The same findings were concluded in a recent study using whole genome analysis (8).

As presented in Table II, it is notable that all the substitutions are tolerated and pathogenic. Of the first four mutations, two are located within domain I that spans aa 313-515 of the EBOV $\mathrm{L}$ protein in the alignment in the present study (aa 222-426 without the gaps for improved alignment). As demonstrated previously, this domain contains stretches of highly conserved residues and motifs, including GHP (9). In a previous study, it was demonstrated that the binding domain for the polymerase cofactor VP35, is located within the first 370 amino acids of the $\mathrm{L}$ protein towards the $\mathrm{N}$-terminal, while the core binding domain is located between aa 280-370. This binding domain overlaps with part of domain I (23). Previous site-directed mutagenesis studies into the Sendai virus $\mathrm{L}$ protein have demonstrated that mutation in this domain leads to a defective replication and transcription mechanism and improper $\mathrm{P}$ protein binding (24). The first four mutations presented in Table II are located within this binding domain, with the M337I and Q346H mutations specifically in the core binding region. However, such mutations appear to be advantageous to the 2014 EBOV and may have enhanced genome replication efficiency. The fourth substitution is isolated, it does not lie within any known functional motif, thus, its role, if any, in the virulence of the virus requires elucidation by site-directed mutagenesis studies.

The next five mutations are also notable. All the mutations lie close to each other within a span of 90 amino acids. These mutations occur outside the conserved domains, within a region of high variability also observed within previous filovirus L protein sequences by comparative analysis (17). Further research regarding this region is required prior to drawing any conclusions about these mutations.

Notably, the S1826D mutation lies within the conserved motif $\mathrm{C}$ identified earlier (17). The mutation is close to the GXGXG pentapeptide. Earlier mutation studies within this region in the Sendai virus have led to reduced replication and transcription activities, however, it did not completely block the polymerase function (25). This mutation is tolerated and appears to be a gain of function mutation. 
The final three mutations lie within a highly unconserved region, for which no functional domains or motifs have been defined or hypothesized. However, the I1951V substitution is notable. The amino acid isoleucine is conserved at position 1,951 in all other Ebolavirus species. It is only substituted to valine in the 2014 EBOV isolates. The impact of this substitution remains to be elucidated and requires further research.

In conclusion, the present study provided an overview of the $\mathrm{L}$ protein amino acid sequences of the $2014 \mathrm{EBOV}$ isolates. The sequence and mutation analysis of L polymerase will aid in advancing towards confinement of future outbreaks. The current study may guide future site-directed mutagenesis studies, and design of site-specific direct targeting antiviral therapeutic agents. It may also help with the development of future diagnostic studies.

\section{References}

1. Sanchez A, Geisbert TW and Feldmann H: Filoviridae: Marburg and ebola viruses. In: Fields Virology. Knipe DM and Howley PM (eds). Lippincott Williams and Wilkins, Philadelphia, PA. pp1409-1448, 2007.

2. Kuhn JH, Becker S, Ebihara H, Geisbert TW, Johnson KM, Kawaoka Y,Lipkin WI, Negredo AI, Netesov SV, Nichol ST, et al: Proposal for a revised taxonomy of the family Filoviridae: Classification, names of taxa and viruses and virus abbreviations Arch Virol 155: 2083-2103, 2010.

3. Sanchez A, Kiley MP, Holloway BP and Auperin DD: Sequence analysis of the Ebola virus genome: Organization, genetic elements, and comparison with the genome of Marburg virus. Virus Res 29: 215-240, 1993.

4. Boehmann Y, Enterlein S, Randolf A and Mühlberger E: A reconstituted replication and transcription system for Ebola virus Reston and comparison with Ebola virus Zaire. Virology 332: 406-417, 2005

5. Waheed Y: Ebola in West Africa: An international medical emergency. Asian Pac J Trop Biomed 4: 673-674, 2014.

6. Waheed Y, Bhatti A and Ashraf M: RNA dependent RNA polymerase of HCV: A potential target for the development of antiviral drugs. Infect Genet Evol 14: 247-257, 2013.

7. Waheed Y, Saeed U, Anjum S, Afzal MS and Ashraf M: Development of global consensus sequence and analysis of highly conserved domains of the HCV NS5B prote in. Hepat Mon 12: e6142, 2012.

8. Gire SK, Goba A, Andersen KG, Sealfon RS, Park DJ, Kanneh L, Jalloh S, Momoh M, Fullah M, Dudas G, et al: Genomic surveillance elucidates Ebola virus origin and transmission during the 2014 outbreak. Science 345: 1369-1372, 2014.

9. Poch O, Blumberg BM, Bougueleret L and Tordo N: Sequence comparison of five polymerases (L proteins) of unsegmented negative-strand RNA viruses: Theoretical assignment of functional domains. J Gen Virol 71: 1153-1162, 1990.
10. Clamp M, Cuff J, Searle SM and Barton GJ: The Jalview Java alignment editor. Bioinformatics 20: 426-427, 2004.

11. Katoh K and Standley DM. MAFFT multiple sequence alignment software version 7: Improvements in performance and usability. Mol Biol Evol 30: 772-780, 2013.

12. Nuin PA, Wang $Z$ and Tillier ER: The accuracy of several multiple sequence alignment programs for proteins. BMC bioinformatics 7: 471, 2006.

13. Tamura K, Stecher G, Peterson D, Filipski A and Kumar S: MEGA6: Molecular evolutionary genetics analysis version 6.0. Mol Biol Evol 30: 2725-2729, 2013.

14. Saitou N and Nei M. The neighbor-joining method: A new method for reconstructing phylogenetic trees. Mol Biol Evol 4: 406-425, 1987

15. Ng PC and Henikoff S. Predicting deleterious amino acid substitutions. Genome res 11: 863-874, 2001.

16. Marston DA, McElhinney LM, Johnson N, Müller T, Conzelmann KK, Tordo $\mathrm{N}$ and Fooks AR: Comparative analysis of the full genome sequence of European bat lyssavirus type 1 and type 2 with other lyssaviruses and evidence for a conserved transcription termination and polyadenylation motif in the G-L 3 ' non-translated region. J Gen Virol 88: 1302-1314, 2007.

17. Volchkov VE, Volchkova VA, Chepurnov AA, Blinov VM, Dolnik O, Netesov SV and Feldmann H: Characterization of the L gene and 5' trailer region of Ebola virus. J. Gen Virol 80: 355-362, 1999.

18. Schnell MJ and Conzelmann KK: Polymerase activity of in vitro mutated rabies virus L protein. Virology 214: 522-530, 1995.

19. Blumberg BM, Crowley JC, Silverman JI, Menonna J, Cook SD and Dowling PC: Measles virus L protein evidences elements of ancestral RNA polymerase. Virology 164: 487-497, 1998

20. Ogino $T$ and $B$ anerjee $A K$ : The $H R$ motif in the RNA-dependent RNA polymerase L protein of Chandipura virus is required for unconventional mRNA-capping activity. J Gen Virol 91: 1311-1314, 2010

21. Ferron F, Longhi S, Henrissat B and Canard B: Viral RNA-polymerases-a predicted 2'-O-ribose methyltransferase domain shared by all Mononegavirales. Trends Biochem Sci 27: 222-224, 2002.

22. Grard G, Biek R, Tamfum JJ, Fair J, Wolfe N, Formenty P, Paweska $J$ and Leroy E: Emergence of divergent Zaire Ebola virus strains in democratic republic of the Congo in 2007 and 2008. J Infect Dis 204 (Suppl 3): S776-S784, 2011.

23. Trunschke M, Conrad D, Enterlein S, Olejnik J, Brauburger K and Mühlberger E: The L-VP35 and L-L interaction domains reside in the amino terminus of the Ebola virus L protein and are potential targets for antivirals. Virology 441: 135-145, 2013.

24. Chandrika R, Horikami SM, Smallwood S and Moyer SA: Mutations in conserved domain I of the Sendai virus L polymerase protein uncouple transcription and replication. Virology 213: 352-363, 1995.

25. Feller JA, Smallwood S, Horikami SM and Moyer SA: Mutations in conserved domains IV and VI of the large (L) subunit of the Sendai virus RNA polymerase give a spectrum of defective RNA synthesis phenotypes. Virology 269: 426-439, 2000. 